\title{
TRADUÇÃO E CIÊNCIA - ALGUMAS CONSIDERAÇÕES
}

\section{Stela Foley*}

RESUMO: O objetivo do artigo é discutir alguns problemas encontrados na tradução de uma série de 13 ensaios de autoria do físico britânico Stephen Hawking, escritos entre 1976 e 1992 e publicados pela Editora Rocco em 1994 sob o título de Buracos negros e Universos bebês. Para tanto, analisamos alguns exemplos mencionados em uma crítica publicada pelo Prof. C. A. dos Santos, da UFRGS, no "Caderno Cultura" do Jornal Zero Hora, em 1995.

Os exemplos analisados demonstram a necessidade de uma cuidadosa revisão em traduções de textos de cunho científico, caso o tradutor não esteja familiarizado com o jargão a ser utilizado. Muitos acreditam que tais textos dependem apenas de fórmulas e cálculos, o que não é verdade, pois a objetividade e precisão do componente lingüístico, o cuidado com o conteúdo e o uso de termos consagrados pela comunidade em questão são essenciais para que o verdadeiro significado do texto original não seja comprometido. O não-cumprimento de tais quesitos prejudica o leitor que não lê o original, aceita a tradução como tal e, conseqüentemente, fica exposto a impropriedades.

UNITERMOS: tradução; ciência; revisão; real significado do texto original.

ABSTRACT: The object of this article is to discuss certain problems encountered in the translation of a series of 13 essays named Black Holes and Baby Universes by the British physicist Stephen Hawking, written between 1976 and 1992, and published by Rocco

* Mestranda do Programa de Estudos Lingüísticos e Literários em Inglês da Universidade de São Paulo. 
Publishers in 1994. In order to do that, we analysed some examples mentioned in a review by Professor C. A. dos Santos, from UFRGS, published in the Zero Hora newspaper in 1995.

The examples that were analyzed confirm the need for careful revision in the translations of scientific texts, when the translator is not familiar with the jargon to be used. Many believe that these types of texts only depend upon formulae and calculations, which is not really the case, because the objectivity and precision of the linguistic component, the accuracy of the content and the use of appropriate terms generally accepted by the scientific community are very important in maintaining the true meaning of the original text. If the above steps are not followed, the reader who has not read the original will accept the translation as such, therefore being exposed to inaccuracy.

KEYWORDS: translation; science; revision; true meaning of the original text.

O papel exercido pela tradução no processo de disseminação científica sempre foi imensurável, sendo passivel de observação desde os estudos da mais antiga das ciências, a Astronomia. A tradução científica existe desde os tempos da Grécia antiga, passando pela civilização árabe, pelo Império Romano e chegando até as épocas Medieval e Moderna. Na realidade, a reconstrução de muitos textos científicos originais perdidos só foi possivel mediante traduções, evidências únicas de tais conteúdos.

A hegemonia na tradução, conceito proposto por Venuti (1998), nada mais é do que uma seleção feita pelos que detêm o poder em cada época sobre quais textos devem ser traduzidos e foi uma das responsáveis pelo desaparecimento de muitos trabalhos científicos que não foram levados a outras culturas. No entanto, na área da Astronomia, pouco se perdeu, provavelmente porque essa ciência foi por muito tempo representativa da filosofia moral e da descrição do universo. Na verdade, foram questões relacionadas às traduções de textos de Astronomia para outras linguas que iniciaram o debate entre tradutores sobre as 
vantagens das diferentes abordagens no processo de tradução científica.

Os trabalhos pioneiros em Física Moderna, inclusive a Teoria da Relatividade de Albert Einstein, foram escritos em alemão. Também os físicos russos escreveram quase que exclusivamente em russo, além de existir um grande volume de trabalhos na área de Física escritos em francês. Entretanto, no contexto contemporâneo, o inglês tornou-se a língua franca na área científica. Assim, trabalhos escritos originalmente em diversos idiomas foram traduzidos para o inglês. Surpreendentemente, não há registro da ocorrência de problemas relacionados à equivalência, à fidelidade, ou à comunicação de significados durante o processo tradutório.

A fácil aceitação das traduções da Teoria dos Quanta e da Relatividade, sem nenhuma menção às dificuldades encontradas durante tal processo, aponta para a pouca valorização que a ciência dá à tradução. Na verdade, percebe-se uma falta de preocupação com a tradução dos textos de conteúdo científico em geral, pois presume-se que neste tipo de texto o conteúdo essencial depende de fórmulas e cálculos e o componente lingüístico não é considerado como fator substancial para a compreensão. Porém, nos textos científicos também existe uma contínua interdependência entre símbolos e linguagem natural, o que portanto exige uma tradução cuidadosa. Quando nos referimos a uma tradução cuidadosa, referimo-nos a uma tradução bemsucedida, onde as teorias, proposições, hipóteses e termos são traduzidos da forma mais exata possivel. A essa exigência, Popovic (1976) deu o nome de "fator de invariância", e tal fator pode ser claramente visualizado no processo de tradução dos textos científicos, onde a precisão e objetividade do conteúdo devem ser enfatizadas. O texto em si, bem como o número de palavras e páginas podem variar, mas não o seu significado. Em outras palavras, o bom tradutor deve ser capaz de exprimir a essência do original.

Com o intuito de facilitar e aperfeiçoar o seu trabalho, os tradutores, de forma geral, buscam encontrar algumas regras básicas para traduzir cada gênero de texto. Leféver e Belloc identificaram respectivamente sete estratégias para traduzir poesia 
e seis regras gerais para traduzir prosa. Entretanto, através de seus estudos, Sarukkai (2004) comprova que, como o texto científico abrange diversas áreas, fica dificil estabelecer parâmetros para sua tradução. Toury (1995) define a tradução científica como uma arte e uma técnica que exigem um conhecimento profundo das culturas e subculturas de cada língua envolvida, de forma que o conhecimento desempenhe a sua função no sistema da lingua-alvo.

Infelizmente, o próprio caráter do discurso científico (presença de fórmulas, linguagem matemática e um tratamento objetivo do tema) trazem à tona a idéia errônea de que a tradução científica é mais fácil do que a literária, o que na verdade não ocorre. A tradução científica exige uma fidelidade muito maior, pois qualquer desvio feito em alguma de suas variantes será considerado uma transgressão por parte do tradutor, o que não procede no caso do texto literário, onde, de certa forma, há maior liberdade de interpretação. Com referência à fidelidade na tradução científica, Rónai (1952) afirma:

Em regra geral, o nível da tradução técnica é mais elevado que o da literária, pelo menos no que diz respeito à fidelidade. Um erro na versão de uma peça de Shakespeare, quando muito, indignará um crítico; mas na de uma bula de remédio ou de um formulário de materiais de construção pode ter conseqüências imprevisiveis.

De acordo com o Instituto de Lingüística de Londres (1994), o tradutor de textos científicos deve possuir conhecimento na área para a qual traduz, imaginação bem desenvolvida para visualizar o equipamento ou processo descrito, capacidade para preencher as lacunas do texto original, senso discriminatório para escolher o termo mais adequado na língua-alvo, habilidade e clareza no uso da língua para a qual traduz, objetividade, precisão e experiência prática em traduzir. O cumprimento dos requisitos acima é de grande importância, pois para o leitor que não lê o original a tradução assume o papel do original. No caso do leitor leigo, o problema se torna ainda mais grave, pois este, na maioria das vezes, não possui a bagagem científica necessária para contestar eventuais erros de conteúdo decorrentes de 
uma tradução inexata, ficando à sua mercê. Assim, quando o tradutor desconhece o tema a ser traduzido e o jargão que lhe é peculiar, é necessário que a tradução passe por uma cuidadosa revisão, feita por pessoas com conhecimento da área, para que a excelência do original não seja comprometida.

Com relação às questões acima, gostariamos de discutir alguns problemas na tradução de uma série de 13 ensaios de autoria do físico britânico Stephen Hawking, escritos entre 1976 e 1992 e publicados pela Editora Rocco em 1994 sob o título de Buracos negros e Universos bebês. Para tanto, utilizamos os exemplos mencionados na crítica escrita pelo Prof. C. A. dos Santos, da UFRGS, publicada no "Caderno Cultura" do jornal Zero Hora, em 1995.

Um dos exemplos mencionados refere-se à primeira teoria da relatividade de Einstein, que em inglês recebeu a denominação de Special Theory of Relativity e é conhecida pela maioria dos fisicos brasileiros e portugueses como Teoria da Relatividade Restrita; denominação semelhante também é usada pelos fisicos franceses. No livro em questão, porém, a expressão foi traduzida como Teoria da Relatividade Especial, equívoco que geralmente acontece quando a tradução é feita por leigos.

A seguir, na página 83 do original, o autor diz: “...the star will generate heat at its center by converting hydrogen into helium”. A tradução publicada foi: “...a estrela gerará calor em seu centro mediante a conversão de oxigênio em hélio". Este erro de atenção poderia ter sido evitado se o tradutor fosse da área, ou se a tradução fosse revisada por um profissional da área, pois eles saberiam que oxigênio não se converte em hélio.

Na página 119, lê-se: "É possivel que o universo primitivo tenha passado pelo que chamamos de uma fase de transição." Porém, no original encontramos: "It is possible that the early universe underwent what is called a phase transition." Há um equivoco na ordem de palavras na tradução de phase transition, que não significa fase de transição, mas transição de fase, fenômeno relacionado com a mudança de estado da matéria. Por exemplo, no caso da água, há uma transição da fase líquida para a gasosa quando ela ferve, e da fase líquida para a sólida quando ela congela. 
Além dos problemas descritos pelo Prof. Santos, outros pontos que podem ser levantados levam em consideração o contexto em que o termo aparece, já que a tradução de um termo pode variar dependendo da área com a qual trabalhamos. A comprovação da escolha do melhor termo através da contextualização e área de atuação foi feita com o auxílio do Corpus WebCorp. Por exemplo, em seu livro, Hawking menciona a constante G Newton's constant of gravity - que é denominada nos livros da área como "constante da gravitação universal", ou "constante gravitacional", mas não como "constante da gravidade de Newton", conforme aparece na página 84 da tradução. Na verdade, o termo gravity causa muita confusão nas traduções. Algumas vezes é traduzido por "gravidade", outras vezes por "gravitação", dependendo do termo com o qual é colocado. Em inglês, por exemplo, pode-se dizer tanto theory of gravitation, como theory of gravity, traduzidos para o português respectivamente como "teoria da gravitação" e "teoria da gravidade". Ao longo do livro existem vários trechos onde se verifica o uso inapropriado dos termos "gravidade" e gravitação". No WebCorp não encontramos nenhuma ocorrência do termo "constante da gravidade de Newton", utilizado pelo tradutor:

\section{Web :}

Sua pesquisa - “constante+gravidade+newton" - não encontrou nenhum documento correspondente.

Encontramos 1 ocorrência para constante gravidade em uma descrição, não em contexto científico:

Web Resultados $\mathbf{1}-\mathbf{1}$ de aproximadamente $\mathbf{2}$ para “constante+gravidade $(0,15$ segundos $)$.

Fazia-se notar pela grande cabeleira e constante gravidade do porte exterior.

Quando saía, os que o encontravam se compraziam em admirar seu passo quase...

www.cfh.ufsc.br/ simpozio/Megahist-filos / Prim-fil/ 0335y316.html - 99k 
Alguns exemplos das muitas ocorrências encontradas para "constante gravitacional" no contexto de Física estão descritos abaixo:

Web Resultados $\mathbf{1}$ - $\mathbf{1 0}$ de aproximadamente $\mathbf{1 0 . 9 0 0}$ para constante+gravitacional

Pela lei da gravitação universal a força de atração gravitacional entre a Terra e a Lua é dada por

\section{$\mathbf{F}=\mathbf{G} \underline{\mathbf{M m}}$ \\ $\mathbf{d}^{2}$}

onde $\mathrm{G}$ é a constante gravitacional, $\mathbf{M}$ é a massa da Terra, $\mathbf{m}$ é a massa da Lua, e d é a distância entre a Terra e a Lua.

www.on.br/site_edu_dist_estelar/site/conteudo/ modulo1/ cap3-forcas-fundamentais/2-forca-gravitacional.html - 11k Calcular a massa da Terra é um problema de solução simples se conhecido o valor da constante gravitacional G.

A primeira lei de Newton diz: matéria atrai matéria na razão direta das massas e na razão inversa do quadrado da distância. Colocando esta lei matematicamente, podemos escrever... www.feiradeciencias.com.br/sala24/24_faqa6.htm - 8k

Na página 76, lê-se: "pano de fundo de microondas", que não faz sentido como resultado da tradução de "background of microwave radiation", pois a expressão usual para os cientistas é "radiação de fundo", ou "radiação cósmica de fundo". Abaixo, apresentamos os números de ocorrências e alguns dos exemplos:

Web Resultados $\mathbf{1}$ - $\mathbf{1 0}$ de aproximadamente $\mathbf{2 2 . 9 0 0}$ para radiação+fundo

Somente após 1965, quando a radiação cósmica de fundo foi observada e medida por Arno Penzias e Robert Wilson, é que a teoria do Big Bang ganhou força na comunidade científica. www.comciencia.br/reportagens/cosmicos/cos04.shtml-30k20 jul. 2005

Toda vida, em nosso planeta, está exposta à radiação cósmica e à radiação proveniente de elementos naturais radioativos exis- 
tentes na crosta terrestre como potássio, césio etc. A intensidade dessa radiação tem permanecido constante por milhares de anos e se chama radiação natural ou radiação de fundo, e provém de muitas fontes.

www.fisica.net/denis / - 4k

Apresentaremos a evolução das medidas na radiação cósmica de fundo em microondas, desde sua primeira medida em 1992, com o satélite...

www.if.usp.br/bifusp/bifold/bif0305.html - 31k

\section{Web}

Sua pesquisa - "pano+fundo+microondas" - não encontrou nenhum documento correspondente.

Apenas por curiosidade, buscamos contextos onde o termo pano de fundo pudesse ser encontrado. O termo foi localizado em sites de turismo, arte e politica:

Cataratas viram pano de fundo em Foz do Iguaçu. PAULA LAGO enviada especial da

Folha de S.Paulo a Foz do Iguaçu (PR) Foz não são só as cataratas,...

\section{www 1.folha.uol.com.br/folha/turismo/noticias /} ult338u4690.shtml - 18k

Traição é pano de fundo em curta sobre sensualidade e mistério por Danilo Fantinel /

www.terra.com.br/diversao/interface/lotus $2 . h t m$

Outro processo que forma o pano de fundo da atual conjuntura é de origem"nacional": a crise do modelo de desenvolvimento implantado no país de 1930 a $1980 . .$. .

www.pt.org.br/site/artigos/artigos_int.asp?cod $=860-51 \mathrm{k}-$ 20 jul. 2005

Na página 88, deparamo-nos com: "mensageiros da energia gravitacional", resultado da tradução de carriers of gravitacional energy. No Brasil, os cientistas usam a expressão "portadores da energia gravitacional". A palavra "mensageiros" não é usual, não é colocada no contexto científico. Entretanto, a palavra "portadores" o é: "portadores de carga elétrica", "portadores de AIDS", "portadores do cromossomo X", "portadores de defi- 
ciências", mas nunca "mensageiros". No WebCorp observamos que "mensageiros da energia" é usual em contextos religiosos e esotéricos, como verificamos no exemplo abaixo:

ORIXAS (Mensageiros da energia) Altar em homenagem aos nossos queridos Orixas

enfeitado de muitas flores e velas coloridas, onde possamos buscar paz,...

is toe.terra.com.br/planetadinamica/altar/site/ lista_altar_pub2.asp?id_user $=671838$ id_altar $=54975$

Na página 98, encontramos: "tamanho da carga elétrica", como tradução para "size of the electric charge". O termo size é aqui usado como a medida ou quantidade de alguma coisa, não como tamanho. Portanto, a forma usual seria: "magnitude da carga elétrica".

Buscando exemplos de ambas as expressões, "magnitude de carga elétrica" e "tamanho de carga elétrica" no WebCorp, encontramos:

Web Resultados $\mathbf{1}$ - $\mathbf{1 0}$ de aproximadamente 364 para magnitude+carga+eletron

Determine: a) a capacitância; b) a magnitude da carga elétrica;... Dados: carga do elétron=1,6×10-19C, massa do elétron=9, $1 \times 10-31 \mathrm{~kg} . .$.

www.fisica.ufop.br/textos/L2EH.doc

O resultado: a carga do elétron "e" é negativa e tem como magnitude 1,60/10.000.000.000.000.000.000 Coulombs. Millikan também determinou que as cargas...

www.ene.unb.br/ene/grad/grad_hist.html - 35k

q: $\underline{\text { magnitude da carga do elétron }}=1,60$ 10-19 C. Região de polarização direta (2)À temperatura ambiente $(20 \circ \mathrm{C}), \mathrm{VT}=25,2$ $\mathrm{mV}$....

\section{www.ene.unb.br/ flavia/ Aulas03-04-DCE-200402 Web}

Sua pesquisa - "tamanho+carga+eletron" - não encontrou nenhum documento correspondente.

A expressão quantum mechanics, com as palavras separadas, foi corretamente traduzida no livro por "mecânica quântica". 
Mas quando essas palavras são unidas por um hífen, quantummechanical - elas não podem ser traduzidas como "mecânicoquântico", como se vê na página 87. A tradução consagrada pela literatura científica é "quantum-mecânico", que exerce a função adjetiva dentro da frase, como observamos no número de ocorrências e exemplo abaixo:

Web Resultados $\mathbf{1}-\mathbf{1 0}$ de aproximadamente $\mathbf{7 9 9}$ para quantum+mecanico $(0,11$ segundos)

O vácuo quantum-mecânico. 4 Ver Também. 5 Referências Externas... O vácuo quantum-mecânico. A Física quântica revela que mesmo um vácuo... encyclopedie-pt.snyke.com/articles / vacuo.html

Por outro lado, o único exemplo encontrado para o termo "mecânico quântico", em um site cômico, refere-se ironicamente a um estudioso da mecânica quântica. É comum dizermos que alguém é um físico, mas não se diz que alguém que estuda mecânica quântica é um mecânico quântico:

Um mecânico quântico é um cara cego num quarto escuro procurando um gatopreto que não existe. No mundo existem 010 tipos de pessoas:...

\section{deagol.blogspot.com/2005_01_02_deagol_archive.html-64k}

Concluindo, podemos reafirmar que a tradução científica produz muitos textos e dissemina a ciência, tendo um papel importante na transmissão do conhecimento. Portanto, o tradutor deve ser suficientemente ético para reconhecer suas limitações ao aceitar um trabalho de tradução e também suficientemente humilde para buscar ajuda durante o processo.

As ferramentas de corpus são extremamente úteis para o tradutor, mas não dispensam uma cuidadosa revisão técnica feita por pessoas com conhecimento na área. Em muitos casos, o tradutor é levado a cometer erros por pura ingenuidade. Por desconhecer a área, ele não possui a malícia necessária para realizar as melhores escolhas dentro do leque de possibilidades que o corpus pode lhe oferecer. Assim, a transferência do conhecimento científico através da tradução multiplica a ciência e 
aumenta a diversidade e funcionalidade de seus textos e discursos; porém, só ocorre se realizada com responsabilidade e ética.

\section{Referências bibliográficas}

HAWKING, S. (1993) Black holes, baby universes and other essay. New York: Bantam.

POPOVIC, A. (1976) A Dictionary for the Analysis of Literary Translation. Edmonton: University of Alberta.

RÓNAI, P(1952) As ciladas da tradução técnica. In: Escola de Tradutores. Rio de Janeiro: Educação e Comunicação Editora Ltda. Educon. SANTOS, C. Buracos Negros, Universos Bebês e outros ensaios - tradução de Maria Luiza X. de A. Borges, Jornal Zero Hora, Caderno 2, 29/7/1995.

SARUKKAI, S. (2002) Translating the world: Science and Language. Lanharn: University Press of America.

VENUTI, L. (1998) The Scandals of Translation. New York and London: Routledge.

TOURY, G. (1995) Descriptive translation studies and beyond. Amsterdam: Benjamins.

\section{Sites:}

Google: http://www.google.com http://www.google.com.br

WebCorp: http:// www.webcorp.com.uk 